\title{
Some New Questions about the Seasonal Decrease of the Ozone Layer
}

\author{
Jaime González Velasco \\ Facultad de Ciencias, Universidad Autónoma de Madrid, Madrid, Spain \\ Email: jaime.gonzalez@uam.es
}

Received March 14, 2012; revised June 21, 2012; accepted July 5, 2012

\begin{abstract}
The depletion-recovery cycle of the ozone layer is explained on the basis of the magnetic properties of oxygen, ozone, nitrogen oxides and chlorine combinations, which upon interaction with the Earth's magnetic field, give rise to gaseous fluxes polar- and equatorial-wards.
\end{abstract}

Keywords: Depletion-Recovery Cycle; Ozone Layer; Magnetic Properties; Oxygen; Ozone

\section{Introduction}

The wide concern awoke in the public opinion by the detection of an ozone hole each spring [1] at latitudes close to the Earth's poles has determined that a high number of scientific observations appear, for which a convincing explanation does not exist yet. Thus, the rate of ozone depletion measured during the polar spring time has been interpreted as a result of the combination of extremely cold temperatures leading to the formation of polar stratospheric clouds, which, together with the presence of nitrogen oxides, chlorine atoms and other chemicals, as well as the beginning of the incidence of photons composing sunlight are able to drive photochemical processes responsible for the ozone depletion.

The fact that the geographic extension of the ozone loss is larger than the surface recognised as perturbed by chemicals, raises doubts about the reason which is generally accepted as the cause of the ozone depletion. Furthermore, ozone losses have been observed even before spring time, i.e., before photons reach polar regions and, as a consequence of it, photochemical degradation reactions can take place [2]. Other unexplained facts are that the rates at which the photochemical processes, supposed to be involved in the ozone depleting mechanisms, are too low to satisfactorily explain the actual rate of ozone decline. In addition to this, the sudden recovery in ozone concentration detected during the spring time is also not yet understood. The commonly accepted interpretation, according to which, the formation-dissipation of the polar vortex is connected with the growth or decrease of the ozone concentration seems to be rather arguable. Measurements have been made trying to correlate ozone concentrations measured at different latitudes, altitudes and year seasons with the abundance of gaseous species able to participate in photochemical reactions supposedly involved in the destruction of the ozone layer (like nitrogen oxides $\left(\mathrm{NO}_{\mathrm{y}}\right)$, chlorine monoxide radicals or chlorine atoms).

The studies made on the evolution with time of the stratospheric concentration of ozone lead to the suspicion that the measured low levels in ozone concentration detected in spring time were due to perturbations in the chemistry of the gases forming part of the Antarctic (or Arctic) stratosphere induced by the large abundances in winter of chlorine radicals and $\mathrm{NO}_{\mathrm{y}}$, which participate in chain photochemical or chemical reactions with $\mathrm{O}_{3}$ molecules. Such reactions are favoured by the cold winter temperatures, by the start of the presence of light photons and by the formation of a third body (like ice needles). In this way, the photochemical processes of ozone decomposition become enhanced, leading to the ozone concentration decline observed [3-7]. Nonetheless, no satisfactory explanation has been given for the moment about the reason of the abundance of nitrogen oxides or chlorine combinations in polar latitudes, i.e., far from the geographic areas where they are most commonly produced.

In this work a new explanation for the depletion-recovery cycles of the ozone layer in polar latitudes is presented based on the interaction between the Earth's magnetic field and gaseous molecules paramagnetic or diamagnetic in nature. Thus, molecules forming part of gaseous substances like $\mathrm{NO}_{\mathrm{x}}, \mathrm{O}_{2}, \mathrm{ClO}_{\mathrm{x}}$, are paramagnetic and upon interaction with the Earth's magnetic field they would be continuously transported from the geographic areas where they are produced by natural or human activities towards the North and South poles following the 
direction of the force lines of the magnetic field. Likewise, the diamagnetic ozone molecules would tend to become shifted by the magnetic field towards equatorial latitudes. Eventually, superimposed to this continuous flux, diffusion and/or convection (winds) driven fluxes may also play a role. During polar winters, the presence of high concentrations of gases formed by paramagnetic molecules, like $\mathrm{NO}_{\mathrm{x}}$ and $\mathrm{ClO}_{\mathrm{x}}$, accumulated by the continuous transport induced by the terrestrial magnetic field, would chemically attack ozone molecules present in those latitudes, significantly depleting its concentration. Furthermore, the rate of the ozone depleting reaction is known to become enhanced by the presence in the atmosphere of little ice needles formed as consequence of the low temperatures and which act as necessary third bodies receiving the excess energy released during the chemical or photochemical reaction of conversion of ozone in oxygen. Therefore, the oxygen concentration would increase during the whole winter time due both, to its formation as a product of the ozone depletion reaction and also as a consequence of the paramagnetic nature of the oxygen molecules and their consequent magnetic induced transport towards the poles. Simultaneously, the ozone concentration becomes depleted without disposing of any mechanism of regeneration, since no photons reach the polar latitudes during the polar night, whose presence could induce the photochemical reactions necessary for the ozone formation. Thus, at the beginning of the spring time some photons reach the polar latitudes and, in the presence of high concentrations of oxygen, a photochemical regeneration reaction of ozone begins, which becomes accelerated the longer the days are and, therefore, the longer the illumination times. Furthermore, at higher temperatures a decrease of the number of ice needles in the air would determine a corresponding decrease of the third body effect and, therefore of the rate of the ozone degradation reactions. In this way, it can be understood the sudden recovery of the ozone layer detected at the beginning of the summer in both poles. The process would compete with photochemical degradation reactions of the ozone layer. In autumn, the illumination times begin to become shorter, so that less and less ozone is formed by photochemical reactions, whereas the chemical destruction reactions of ozone continue, explaining the new decrease of ozone concentrations measured at that time.

In summary, according to the arguments expressed above, the ozone layer is submitted, in polar latitudes, to annual depletion-recovery cycles, which can be explained as consequence of transport mechanisms of gases formed by molecules paramagnetic and diamagnetic in nature. If this were the cause of the ozone cycle detected, any influence of solar winds on the local intensities of the Earth's magnetic field should produce corresponding variations in the formation and degradation's rate of ozone. In general, any variation measured in the Earth's magnetic field, whatever would provoke it, should lead to measurable effects on gases transported polar- or equatorial-wards.

\section{Correlation between the Concentrations of Nitrogen Oxides and Ozone at Different Latitudes and Altitudes}

Some authors have published studies giving the rate of change of the ratio $\mathrm{O}_{3} / \mathrm{NO}_{y}$ measured at different latitudes and altitudes [8] The $\mathrm{O}_{3} / \mathrm{NO}_{\mathrm{y}}$ ratio decreases from northern to southern latitudes in the austral hemisphere, until reaching a latitude boundary placed about $66^{\circ} \mathrm{S}$. At more southern latitudes than the above cited boundary an increase of the $\mathrm{O}_{3} / \mathrm{NO}_{\mathrm{y}}$ ratio was detected, the closer to the South Pole the measurements were made. Such an unexpected behaviour has been interpreted as the consequence of the high degree of denitrification taking place within this region. The general trend for the change of the $\mathrm{O}_{3} / \mathrm{NO}_{\mathrm{y}}$ ratio with the shift towards more southern latitudes is in agreement with a transport of paramagnetic $\mathrm{NO}_{\mathrm{y}}$ molecules along the magnetic line forces and towards places where the magnetic intensity is higher. This magnetically induced transport is steadily taking place contributing to accumulate paramagnetic gases molecules near polar latitudes during the whole year. Such a magnetic effect would prevail over the levelling up effect which would be exerted on any gas distribution by a transport driven mainly by atmospheric fluxes or by diffusion. The decrease in the $\mathrm{O}_{3} / \mathrm{NO}_{\mathrm{y}}$ ratio could not be attributed to transport or mixing of air vertically nor horizontally and was initially explained as caused by some unknown chemical mechanism. In addition to this, the minimum value of the ratio was found in the proximity of the magnetic pole, as it could have been predicted using as argument the magnetic interactions already mentioned.

Similarly, measurements of the $\mathrm{O}_{3} / \mathrm{NO}_{\mathrm{y}}$ ratio [8] indicate that it slightly increases with the altitude, which is again in agreement with a shift of the paramagnetic $\mathrm{NO}_{\mathrm{y}}$ molecules towards lower altitudes, where the intensity of the magnetic field is higher. Therefore, both trends of variation of the concentration ratio can be rationalized by taking into account the mentioned magnetic interaction.

On the other hand, the $\mathrm{NO}_{\mathrm{y}}$ concentration seems to remain unperturbed relative to that of $\mathrm{N}_{2} \mathrm{O}$, when parallel measurements of the concentration of both substances were carried out. In the lower atmosphere a persistent linear negative relationship between the concentrations of $\mathrm{N}_{2} \mathrm{O}$ and $\mathrm{NO}_{\mathrm{y}}$ was found, which could be fitted [8] to the following linear equation: $\left[\mathrm{NO}_{\mathrm{y}}\right]=20800-69.8 \times$ $\left[\mathrm{N}_{2} \mathrm{O}\right]$.

This linear relationship was interpreted as the result of a balance of photochemistry, transport and mixing of 
gases. In this case, $\mathrm{NO}_{\mathrm{y}}$ is formed by paramagnetic molecules whereas $\mathrm{N}_{2} \mathrm{O}$ molecules are diamagnetic, which could also explain the cited negative relationship.

\section{Contradictory Questions on the Interpretation of the Ozone Hole}

Almost any of the above cited studies [3-7] coincide in attributing the seasonal and annual variability in the ozone concentration to mechanisms in which chemical reactions in the gas phase intervene, where photochemical induced processes play a fundamental role. However, experimental data obtained on the kinetics of any of the photochemical processes suspected to be taking place present values for the reaction rates far below those which should be necessary in order to give an account of the seasonal changes in stratospheric ozone concentrations in latitudes close to the poles.

Some apparently contradictory questions remain yet open to discussion, like: why the maximum amount and rate of ozone destruction takes place at polar latitudes, i.e., far away from the geographic zones where a maximum human activity is located and, therefore, where a maximum amount and concentration of potentially dangerous gases are produced. Such issues are unquestionably linked with the fact that measurements detect in polar latitudes maximum concentrations of $\mathrm{ClO}$ and of active nitrogen oxides $\left(\mathrm{NO}_{\mathrm{y}}\right)$ just at the beginning of the spring time, which is the time at which a maximum destruction rate of the ozone layer is observed.

Furthermore, it is also known that the ozone hole formed at Arctic polar latitudes is smaller than that appearing at the Antarctic ones, notwithstanding the evidence that the concentrations of chlorofluorocarbons and nitrogen oxides should be higher in the polar latitudes of the northern hemisphere than those present in polar latitudes corresponding to the southern one, due both, to a more dense accumulation of industrial activities, as well as to a more intense aerial traffic in the north than in the south, with the consequence of the formation of higher amounts of active nitrogen oxides in combustion engines and fuselages of aircrafts.

Another controversial question is how to interpret the sudden recovery of the ozone concentration detected every summer in polar latitudes. One attempt of understanding is to attribute it to a dissipation of the Antarctic polar vortex formed before winter. Doubts about that interpretation are sustained by the short time during which such a recovery takes place and mainly by the fact that the area trapped in the Antarctic polar vortex extends over the $66^{\circ}$ of southern latitude, whereas the chemically perturbed zone where the maximum destruction rate of ozone is observed scarcely reaches the $60^{\circ}$ of southern latitude.

\section{The Earth's Magnetic Field and the Magnetic Properties of Oxygen, Nitrogen Oxides, Chlorine Atoms and Some Chlorine Combinations and Ozone Molecules}

Many of the problems cited above can be overcome by taking into account an effect which should be playing an important role in the transport of gaseous substances throughout the atmosphere. In the analysis of the data made until the moment an important physical property of gaseous molecules has not been considered. This property is linked to the presence in the HOMO of the concerned molecule of paired or unpaired electrons. In the first case the corresponding molecules would be diamagnetic in nature, whereas in the second instance they would be paramagnetic. The diamagnetic molecules interact with a magnetic field in such a way that they would tend to be driven towards regions where the magnetic field intensity is lower, unlike the paramagnetic ones, which would be shifted towards places where the magnetic field intensity is higher. Thus, for the case of the Earth's magnetic field, paramagnetic molecules would be driven towards the poles where the magnetic field presents maximum intensities, whereas diamagnetic molecules would tend to be shifted from polar latitudes towards equatorial ones.

A classification can be made of the gaseous substances which participate in the chemical and photochemical reactions of destruction of the ozone layer. Thus, paramagnetic species are $\mathrm{O}_{2}, \mathrm{NO}, \mathrm{NO}_{2}, \mathrm{NO}_{3}, \mathrm{NO}_{\mathrm{y}}, \mathrm{ClO}, \mathrm{Cl}$, whereas diamagnetic species are $\mathrm{O}_{3}$ and $\mathrm{N}_{2} \mathrm{O}$.

The interaction between diamagnetic and paramagnetic gas molecules with the Earth's magnetic field can contribute to a continuous flow of paramagnetic gases towards the poles and of gases composed by diamagnetic molecules equatorial- or tropical-wards. Therefore, according to the classification made before of the magnetic properties of different gaseous molecules present in the atmosphere, $\mathrm{O}_{2}, \mathrm{NO}, \mathrm{NO}_{2}, \mathrm{NO}_{3}, \mathrm{NO}_{\mathrm{y}}, \mathrm{ClO}$ and $\mathrm{Cl}$ tend to accumulate near the poles where they react with the $\mathrm{O}_{3}$ molecules through chemical (in the absence of photons) or photochemical reactions, whenever the conditions are adequate for it (low temperatures which allow the presence of ice needles in suspension able to contribute to the third body effect). Such an accumulation is in agreement with the measured continuous decrease of the $\left(\mathrm{O}_{3} / \mathrm{NO}_{\mathrm{y}}\right)$ ratio mentioned before. The destruction mechanism of ozone is accompanied by the tendency of the ozone molecules to be shifted by the magnetic field towards equatorial latitudes, so that both effects can account for the rapid degradation of the ozone layer observed during the winter.

An attempt of calculation of the rate of displacement 
of gaseous molecules due to magnetic interactions can be made by equalizing the magnetic force, $F_{m}$, acting on individual molecules with the viscous force, $F_{v}$, acting on the molecules due to their displacement in a fluid characterized by its viscosity.

In order to deduce an equation for the magnetic force it is possible to use the equation for the energy of interaction of an electron, $E_{m}$ with a magnetic field of intensity B measured in gauss. This Equation is [9]:

$$
E_{m}=g \mu_{B} \mu_{S} B
$$

where $g$ represents the Landé adimensional factor, which has a value of 2 for an unpaired electron, $\mu_{S}$ the spin magnetic dipole moment, whose value is $\pm 1 / 2, B$ is the magnetic field intensity in gauss and $\mu_{B}$ represents the Bohr magneton, given by following expression:

$$
\mu_{B}=\frac{e h}{4 \pi m_{e} c}=9.273 \times 10^{-21} \frac{\mathrm{erg}}{\text { gauss }}
$$

where $e$ is the electron electric charge, $h$ the Planck constant, $m_{e}$ the electron mass and $\mathrm{c}$ the velocity of light.

The intensity of the magnetic field grows from equatorial to pole regions. Consequently, a magnetic field gradient along the meridians can be defined as $\mathrm{dB} / \mathrm{d} x$ (measured in gauss $/ \mathrm{cm}$ ), which induces a movement of paramagnetic gas molecules towards the poles, where the magnetic field intensity is a maximum. Likewise, this magnetic field gradient induces a movement of diamagnetic gas molecules towards equatorial regions.

Therefore, the magnetic force acting on a paramagnetic gas molecule can be given by the following expression:

$$
F_{m}=g \mu_{B} \mu_{S}\left(\frac{\mathrm{d} B}{\mathrm{~d} x}\right)
$$

where $F_{m}$ is expressed in din, when $\mu_{B}$ is given in erg/gauss and $\mathrm{d} B / \mathrm{d} x$ in gauss $/ \mathrm{cm}$.

On the other hand, the viscous force exerted on the gaseous molecules, supposing they are spherical with a radius $r$, can be described by means of the Stokes Equation [10]:

$$
F_{v}=6 \pi r \eta v
$$

where $r$ represents the Stokes radius of the gas molecules, which are supposed to be spherical, $\eta$ the dynamic viscosity of the atmosphere at the altitude studied and $v$ is the velocity of displacement of the gaseous molecules.

In equilibrium, both forces would become equal to each other. Equalizing the expressions 1 and 2, the velocity of displacement of the gases can be obtained according to following Equation:

$$
v=\frac{g \mu_{B} \mu_{S}\left(\frac{\mathrm{d} B}{\mathrm{~d} x}\right)}{6 \pi r \eta}
$$

Although the magnetic force can be calculated to be very weak, the value of $\eta$ is also rather low at stratospheric altitudes so that the velocity would stabilize at reasonable values. On the other hand $\eta$ would also decrease with increasing values of height, since the density of the atmospheric layer is an exponentially decreasing function of the distance to the Earth surface. An estimation of the magnetic field gradient can be done by taking into account that the strength of the magnetic field is around three times higher in polar than in equatorial latitudes. In this way it can be understood that paramagnetic gas molecules tend to become accumulated in polar latitudes. In a similar way, the gas distribution with the altitude agrees with the fact that the magnetic field strength becomes lower with increasing altitudes.

\section{A Proposition for an Ozone Annual Cycle}

Under the supposition that the Earth's magnetic field is playing a role in the transport mechanism of gases in the stratosphere, an attempt can be made for understanding the depletion-recovery cycles of the ozone layer. Thus, oxygen would be produced at a maximum rate in equatorial and tropical latitudes, where the photosynthetic cycles mediated by the high accumulations of algae in the oceans, or trees and plants contribute to enhance oxygen production, which, on the other hand, is a continuous process over the whole year. The appreciable concentration of oxygen molecules, together with the diary supply of photons provided by solar illumination and specially those of $\lambda<242 \mathrm{~nm}$, enhance the oxygen production of $\mathrm{O}\left({ }^{3} \mathrm{P}\right)$ and, consequently, of ozone molecules, according to the following known reactions [11]:

$$
\begin{gathered}
\mathrm{O}_{2}+h v(\lambda<242 \mathrm{~nm}) \rightarrow 2 \mathrm{O}\left({ }^{3} \mathrm{P}\right) \\
\mathrm{O}\left({ }^{3} \mathrm{P}\right)+\mathrm{O}_{2}+\mathrm{M} \rightarrow \mathrm{O}_{3}+\mathrm{M}
\end{gathered}
$$

where $\mathrm{M}$ is a third molecule which absorbs a part of the energy liberated in the ozone formation. $M$ can be a nitrogen molecule or even another oxygen molecule, which plays the role of a third body absorbing the excess energy released during the process of ozone formation.

The rate of production of ozone would be a maximum in tropical and equatorial latitudes, where a maximum concentration of $\mathrm{O}_{2}$ is produced by photosynthesis and solar photons are found every day during the whole year.

Oxygen molecules would also be produced at any other latitude, but in smaller amounts than there where plants and algae are more abundant. Since $\mathrm{O}_{2}$ molecules are paramagnetic, once formed they would be submitted to a magnetic force giving rise to a shift polar-wards. The number of photons reaching the unit surface area at geographic regions located at intermediate latitudes between the equator and the poles depends on the year's season as well as on the own latitude considered. At the poles, dur- 
ing winter, no photons would reach the atmosphere, so that the mechanism of ozone recovery cannot take place even although the concentration of oxygen would continuously grow due to the magnetic induced transport mechanism. In addition to the fact that ozone is not produced there where photochemical reactions are not possible, the depletion mechanism of ozone continues, since chemical reactions would take place at the atmosphere between the ozone molecules and the nitrogen and chlorine combinations, equally concentrated in the poles due to their paramagnetic nature. On the other hand, ozone molecules, due to its diamagnetic nature, tend to continuously be shifted equatorial-wards by the magnetic field. Both reasons contribute to the decrease of the ozone layer observed in polar latitudes after winter time.

At the beginning of the spring time, two circumstances concur in starting the recovery of the ozone concentration in the poles: 1) a high concentration of oxygen produced by chemical attack of ozone by nitrogen or chlorine combinations (reactions which are favoured by the paramagnetic nature of the final product, oxygen, in those places where the magnetic field strength is higher); and 2) by magnetic induced transport, and the beginning of the illumination which allow photochemical recovery reactions to start. The continuously growing illumination times at the beginning of the spring, together with a maximum concentration of oxygen starts a rapid mechanism of regeneration of the ozone layer. Simultaneously, the temperature increase would lead to a decrease in the amount of ice needles in the stratosphere whose presence is thought to be necessary in order to dissipate the excess energy produced during the ozone degradation reactions, so that also these reactions become partially inhibited.

In autumn, a combination of descending temperatures and decreasing illumination times would give rise to an inhibition of the ozone regeneration mechanism, whereas the atmospheric chemistry conducing to its destruction becomes enhanced by the formation of ice needles.

The ideas above expressed are able to give an account of the ozone depletion-recovery cycles, as well as of the facts that the concentration measured for paramagnetic substances, like chlorine monoxide and that the $\mathrm{O}_{3} / \mathrm{NO}_{\mathrm{y}}$ ratio changes with altitude and latitude in the way predicted by taking into account magnetic interactions.

\section{Conclusions}

Taking into account the paramagnetic or diamagnetic properties of oxygen, ozone, nitrogen oxides, chlorine and chlorine combinations, it is possible to give a coherent account of the seasonal, latitude and altitude variations in the ozone concentration.

In order to verify that such a magnetic effect is actually playing a role it could be interesting to try to corre- late eventual changes in the magnetic field strength produced during the year at different latitudes with variations in the rates of the depletion-recovery cycle of the ozone.

Furthermore, changes in the magnetic field strength could also have an influence on the equilibriums of chemical or photochemical reactions participating in those mechanisms, as well as on the activation enthalpies and, therefore, in the values of the rate constants of the reactions involved. Such effects should be present, whenever reactants, activated complexes and reaction products are of paramagnetic or diamagnetic nature.

\section{REFERENCES}

[1] J. C. Farman, B. G. Gardiner and J. D. Shanklin, "Large Losses of Total Ozone in Antarctic Reveal Seasonal $\mathrm{ClO}_{\mathrm{x}} / \mathrm{NO}_{\mathrm{x}}$ Interaction," Nature, Vol. 315, No. 6016, 1985, pp. 207-210. doi: $10.1038 / 315207 \mathrm{a} 0$

[2] J. M. Rosen, D. J. Hofmann, J. W. Harder and S. R. Rolf, "Ballon/Borne Observations of the Development and Vertical Structure of the Antarctic Ozone Hole in 1986," Nature, Vol. 326, No. 6008, 1987, pp. 59-62. doi: $10.1038 / 326059 \mathrm{a} 0$

[3] D. J. Wuebbles, S. Salomon, R. R. Garcia and F. S. Rowland, "On the Depletion of the Antarctic Ozone," Nature, Vol. 321, No. 6072, 1986, pp. 755-758. doi: $10.1038 / 321755 \mathrm{a} 0$

[4] J. A. Logan, M. B. McElroy, R. J. Salawitch and S. C. Wofsy, "Reductions of Antarctic Ozone Due to Synergistic Interactions of Chlorine and Bromine," Nature, Vol. 321, No. 6072, 1986, pp. 759-762. doi:10.1038/321759a0

[5] F. Arnold and P. J. Crutzen, "Nitric Acid Cloud Formation in the Cold Antarctic Stratosphere: A Major Cause for the Springtime 'Ozone Hole'," Nature, Vol. 324, No. 6098, 1986, pp. 651-655.

[6] A. Parrish, J. W. Barrett, R. M. Solomon, R. L. de Zafra, M. Jaramillo and L. Emmons, "Formation of the Antarctic Ozone Hole by the ClO Dimer Mechanism," Nature, Vol. 336, No. 6198, 1988, pp. 455-458. doi: $10.1038 / 336455 \mathrm{a} 0$

[7] Y. L. Young, S. P. Sander and R. R. Friedl, "Rate of Formation of the ClO Dimer in the Polar Stratosphere. Implications for Ozone Loss," Science, Vol. 245, No. 4922, 1989, pp. 1095-1098. doi:10.1126/science.245.4922.1095

[8] A. F. Tuck, M. H. Proffitt, D. W. Fahey and K. K. Kelly, "High Altitude Ozone Loss Outside the Antarctic Ozone Hole," Nature, Vol. 342, 1989, pp. 233-237. doi: $10.1038 / 342233 \mathrm{a} 0$

[9] G. W. Castillan, "Physical Chemistry," Addison-Wesley Publishing Company, Inc., Reading, 1971.

[10] R. A. Robinson and R. H. Stokes, "Electrolyte Solutions," Butterworths Publications, Ltd., London, 1955.

[11] T. G. Spiro and W. M. Stigliani, "Chemistry of the Environment," Pearson Prentice Hall, Upper Saddle River, 2004. 